Faculty of Veterinary Medicine, Al-Baath University, Syria.

\title{
COMPARATIVE EFFICIENCY OF HERBAL* AND SYNTHETIC METHIONINE ON PERFORMANCE AND IMMUNITY IN BROILERS
}

(With 9 Tables and 3 Figures)

By

\section{A. ABDALLAH and R. ALMONAJED}

(Received at 19/3/2011)

مقارنة تأثثر المثيونين* النباتي والاصطناعي على الكفاعة الإنتاجية

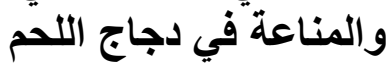

\section{عباس عبل الله ، رياض المنجب}

تم إجر اء تجربة لتقدير إمكانية استخدام المثيونين النباتي بدلا من المثيونين الاصطناعي

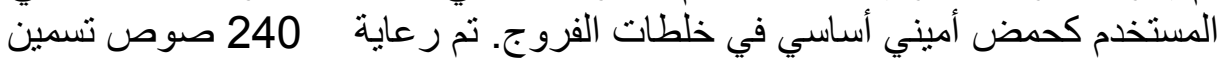

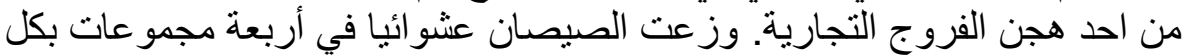

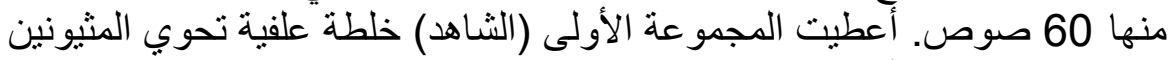

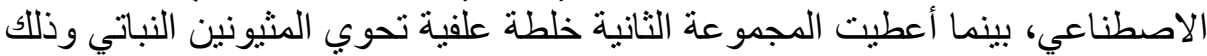

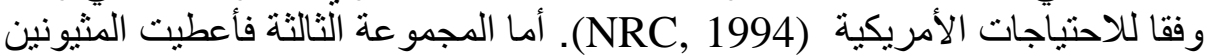

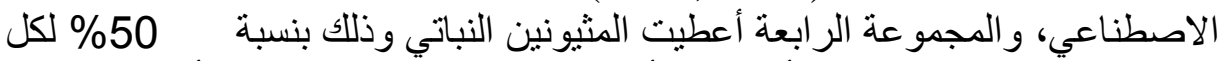

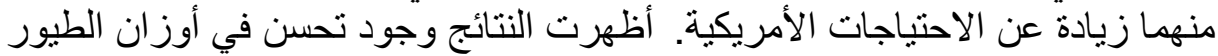

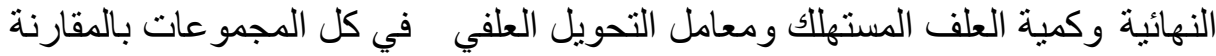

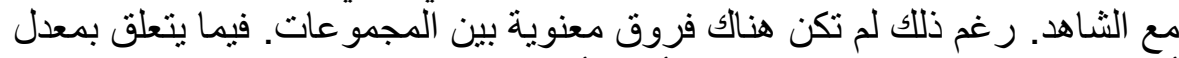

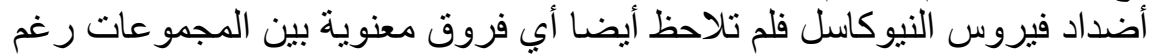

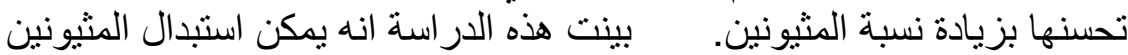

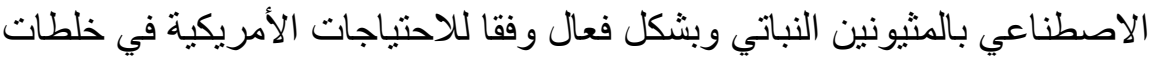

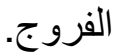

FCR الكلمات المفتاحية :المثيونين النباتي ، المثيونين الاصطناعي ، الكفاءة الإنتاجية ، الفروج F *

\section{SUMMARY}


An experiment was conducted to determine the potential of using herbal methionine to replace DL-methionine as an essential amino acid supplement in broiler diets. Two hundred and forty (240) day-old commercial broiler chicks were randomly divided into four groups, each group consisted of 60 chicks. The birds of groups 1 and 2 were fed an DL-Methionine and Herbal Methionine respectively according to (NRC, 1994) requirements. While groups 3 and 4 DL-Methionine and Herbal Methionine were added at level of $50 \%$ more than (NRC, 1994) requirements. The results showed an improvement in the final body weight, feed intake and feed conversion ratio (FCR) in all groups when compared with the control group, Without any significant differences between groups. The HI-Ab titer of Newcastle disease virus was also did not differ significantly in all groups, inspite of the excess methionine. This study demonstrates that herbal methionine could replace DLmethionine efficiently when used in diets of commercial broiler chicken.

Key words: Herbal methionine, DL-methionine, Performance,Broiler, FCR.

* Herbal Methionine: Herbal ingredients replacing synthetic Methionine.

\section{INTRODUCTION}

$$
\text { المقدم-ة }
$$

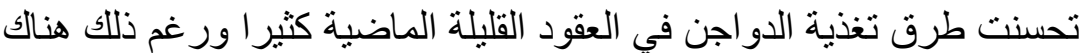

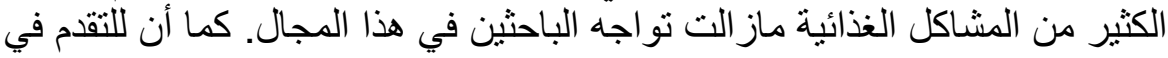

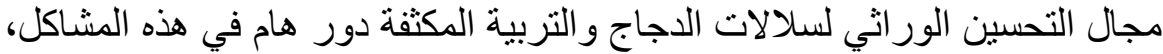

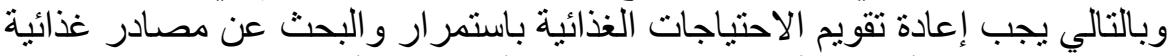

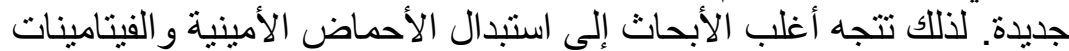

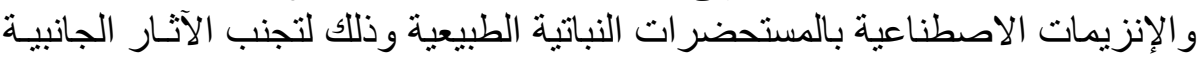

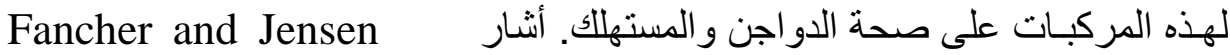

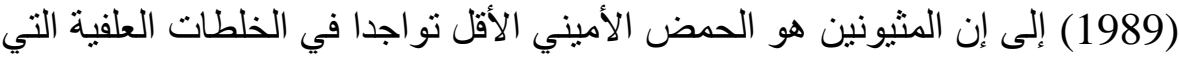

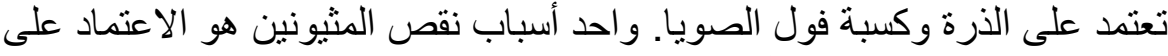

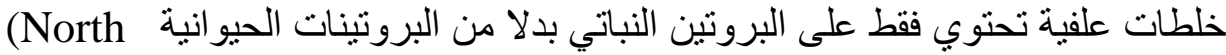

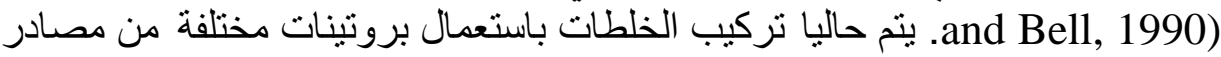

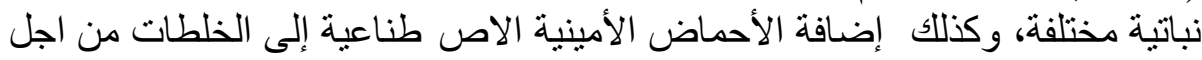

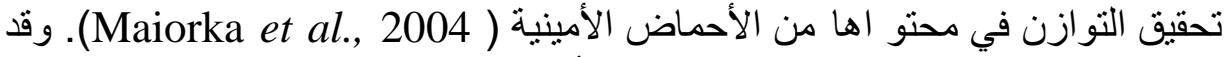

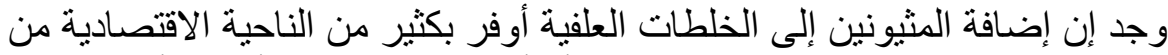

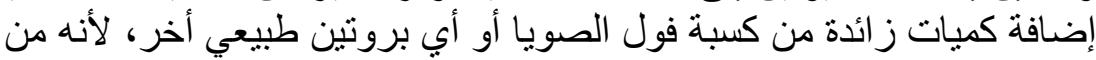

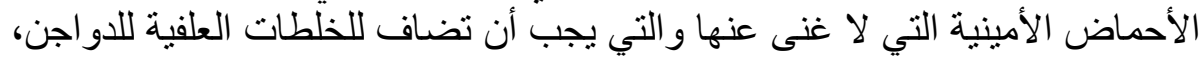


حيث أن الطيور غير قادرة على تركيب الكميات اللازمة منه لاستمر ار الحياة والنمو.

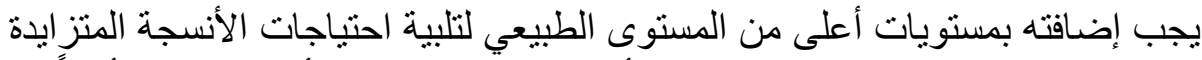

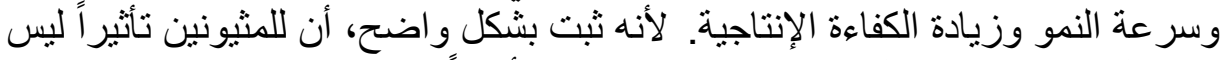

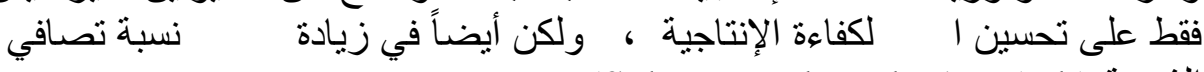
الذبيحة (Schutte and Pack, 1995a,b).

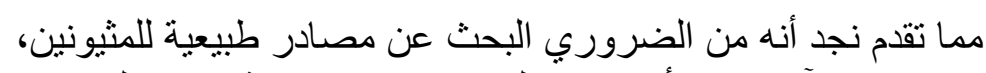

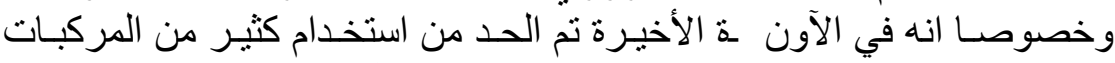

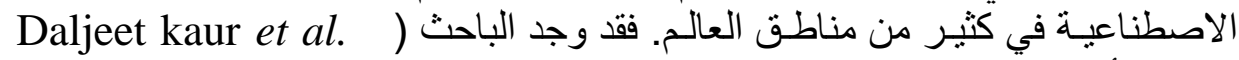

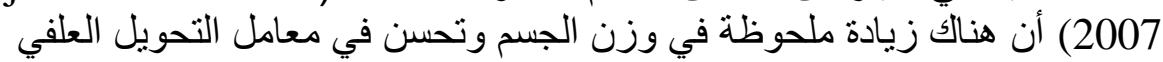

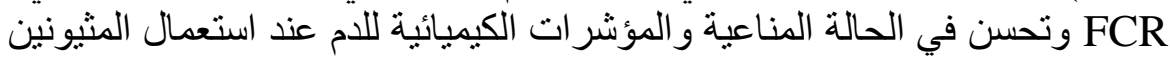

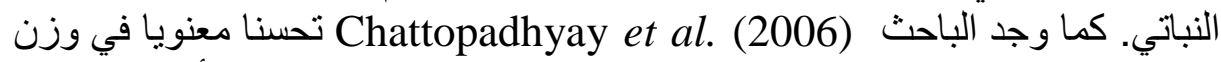

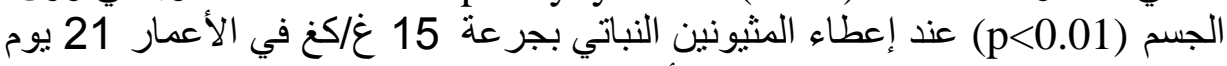

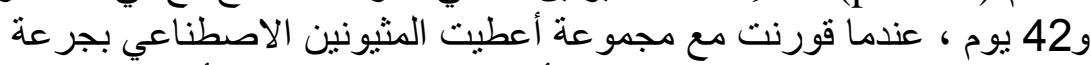

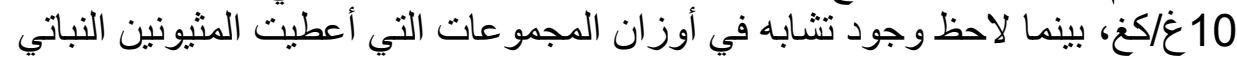

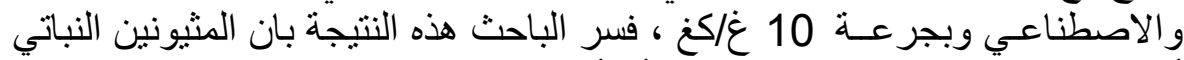

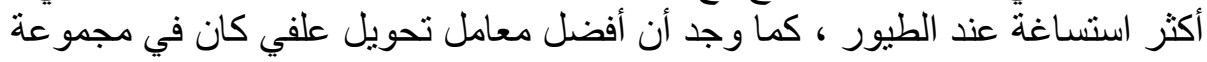

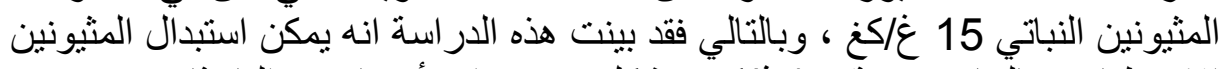

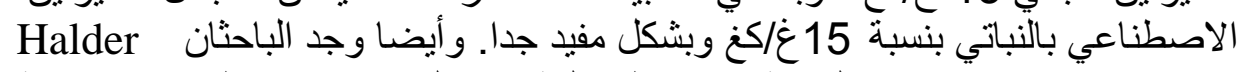

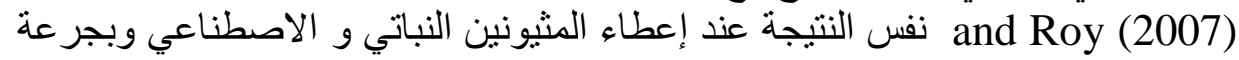

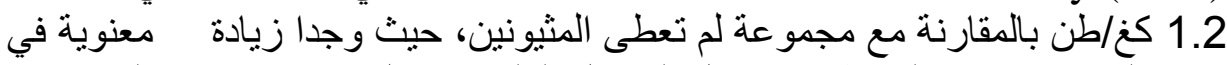

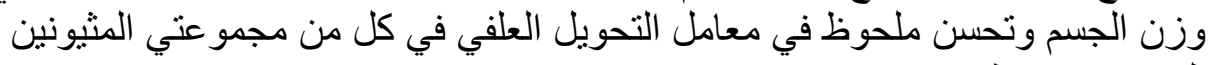

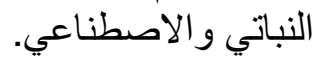

يمكن أن يؤثر نقص الأحماض الأمينة على الاستجابة المناعية حسب رأي الأي

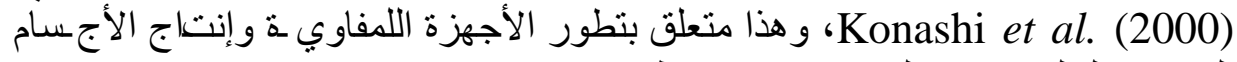

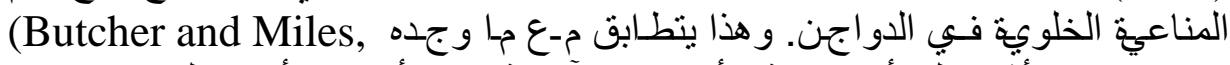

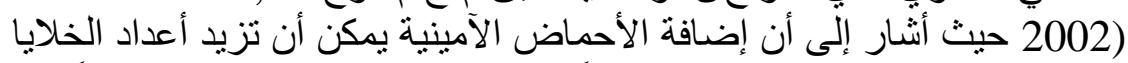

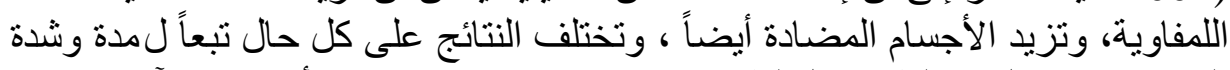

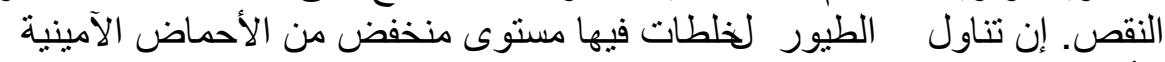

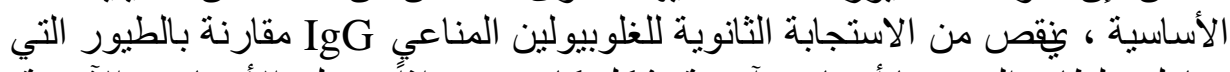

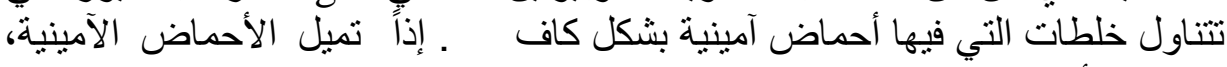

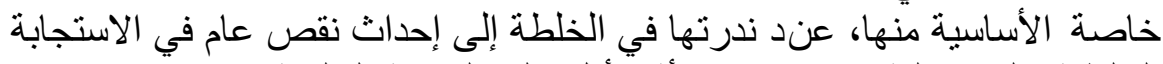

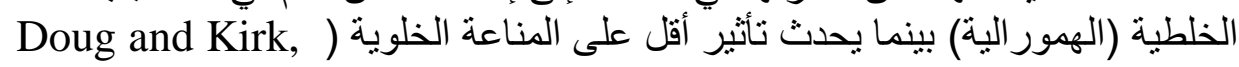
.(2004

أهداف البحث Research Objectives: 
دارسة تأثير المثيونين النباتي مقارنة مع المثيونين الاصطناعي على الكفاعة الكاء

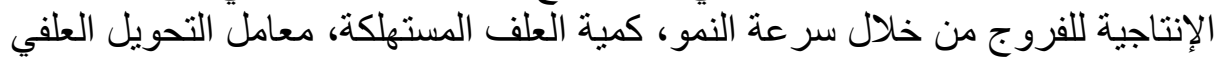
ونسبة النفوق وذذلك بمستو خيين مختلفين:

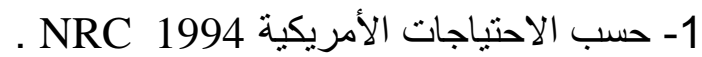
2- أعلى من الاحتياجات الأمريكية بنسبة 50 \% \%

\section{MATERIALS and METHODS مواد وطرائق البحث}

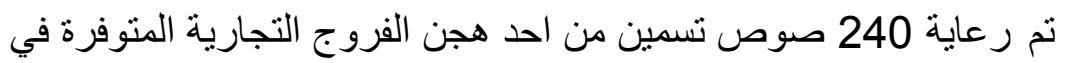

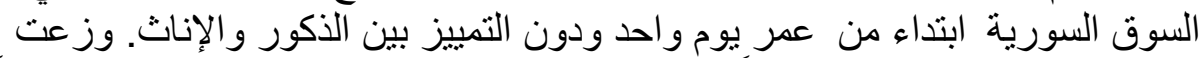

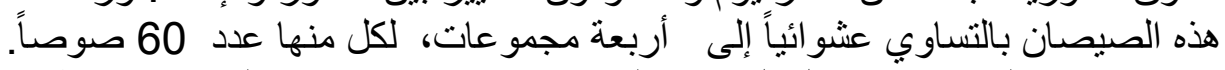

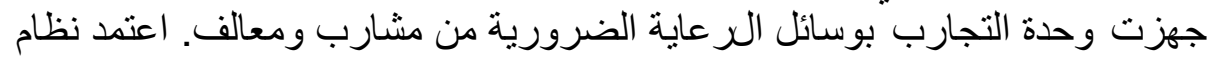

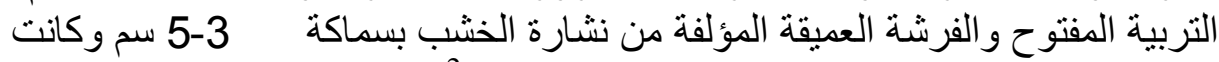

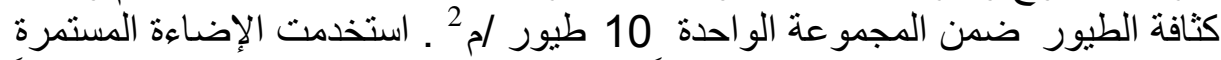

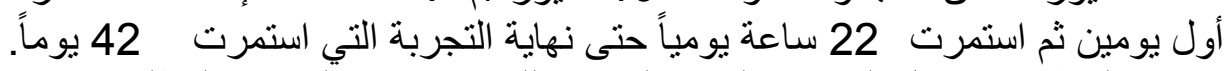

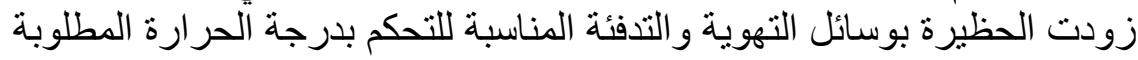

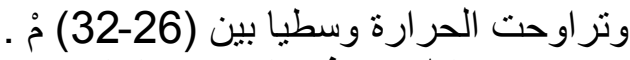

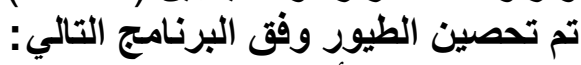

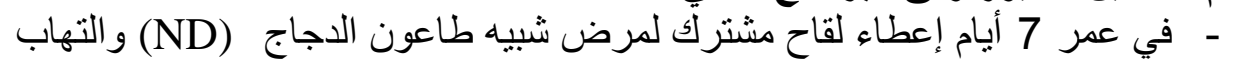

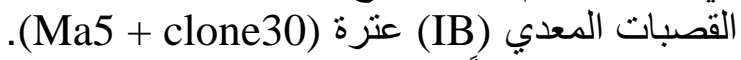
- في عمر 14 يوماً إعطاء لقاح لمرض الجمبورو عترة متوسطة الضراوة بماء

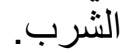
- في عمر 21 يوماً إعطاء لقاح لمرض شبه الطاعون /عترة كلون/ قطرة بالعين

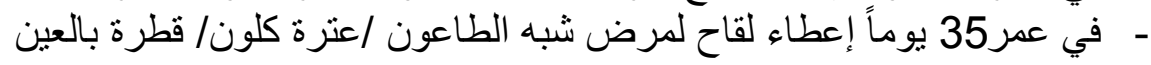

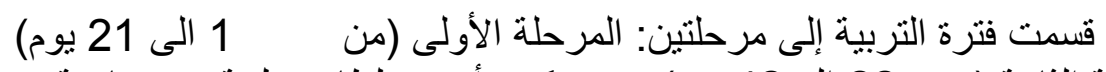

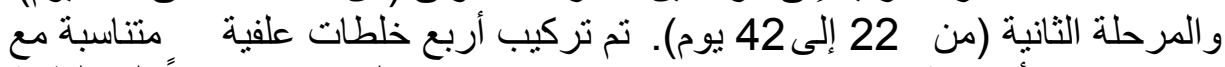

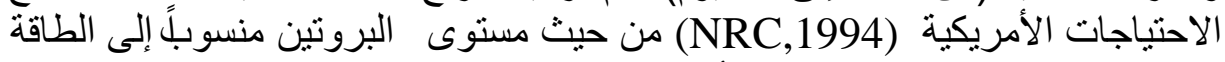

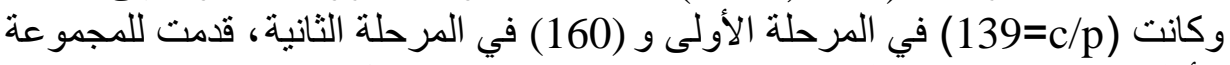

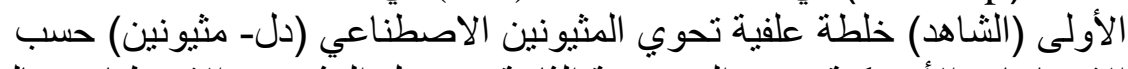

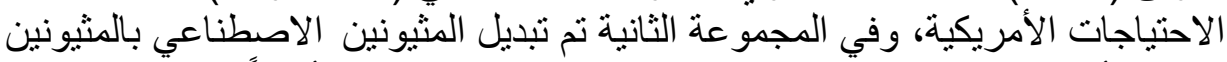

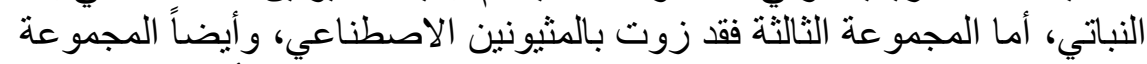

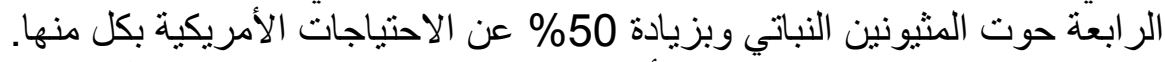

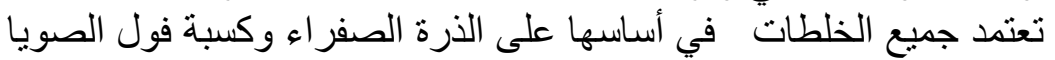

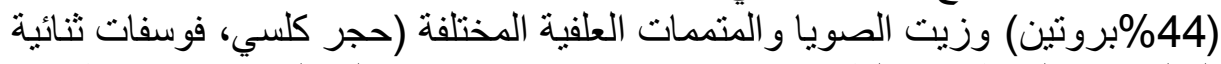

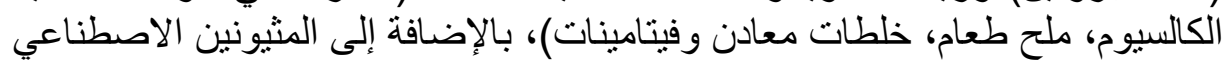




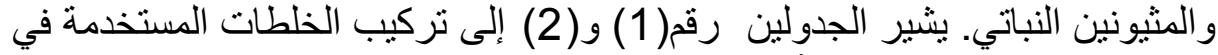

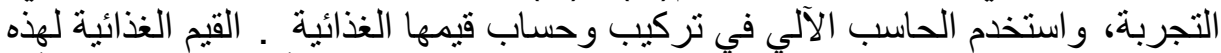
الخلطات موضحة بالجد ولين رقم (3) و و (4). تم تقديم العلف يو مياً بطريقة حرة وفقاً

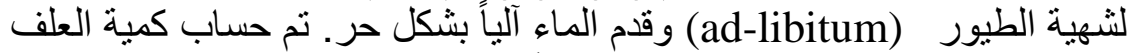

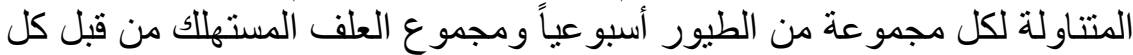

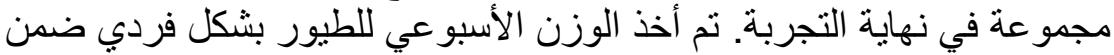

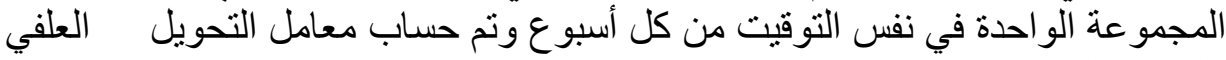

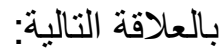

معامل التحويل العلفي= كمية العلف المستهلك بالغرام / الوزن الحي للطائر بالغرام

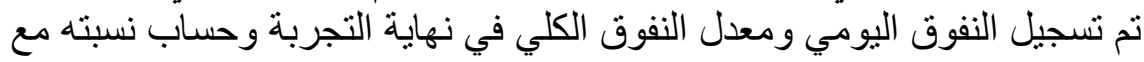

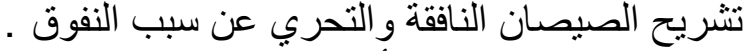

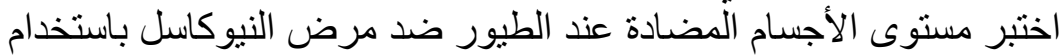

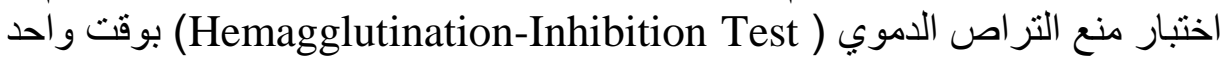
حسب طريقة ( OIE Manual,2000) وطريقة (Lu, 2007).

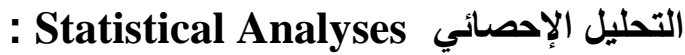

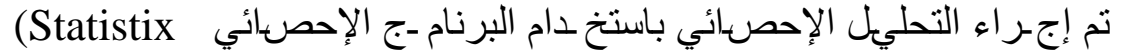

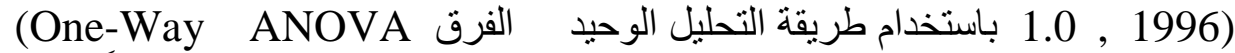
Analysis Of Variance)

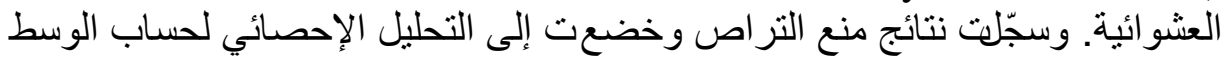
الهندسي لمعيار الأجسام المضادة.

\section{RESULTS}

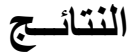

جدول رقم 1: تركيب الخلطات العلفية في المرحلة الأولى

\begin{tabular}{|l|l|l|l|l|}
\hline مثيونين & مثيونين نيونين & & \\
\hline
\end{tabular}


Assiut Vet. Med. J. Vol. 57 No. 130 July 2011

\begin{tabular}{|c|c|c|c|c|}
\hline 2 & الاصطناعي 2 & 1 & الاصطناعي 1 & مكو نات الخلطة \\
\hline 58.29 & 58.25 & 58.3 & 58.3 & ذرة صفر اء \\
\hline 36.4 & 36.34 & 36.5 & 36.5 & كسبة الصويا \\
\hline 1.15 & 1.15 & 1.15 & 1.15 & زيت الصويا \\
\hline 2 & 2 & 2 & 2 & فوسفات ثنائية الكالسيوم \\
\hline 1 & 1 & 1 & 1 & كربونات الكالسيوم \\
\hline 0.32 & 0.32 & 0.21 & 0.21 & مثيونين حر \\
\hline 0.21 & 0.32 & 0.21 & 0.21 & كولين 60\% \\
\hline 0.1 & 0.1 & 0.1 & 0.1 & خلطة فيتامينات* \\
\hline 0.1 & 0.1 & 0.1 & 0.1 & خلطة معادن * \\
\hline 0.43 & 0.43 & 0.43 & 0.43 & ملح طعام \\
\hline 100 & 100 & 100 & 100 & المجموع \\
\hline
\end{tabular}

(*) 1 كل من العلف الجاهز يحقي على الفيتامينات و المعادن النادرة الآتية:

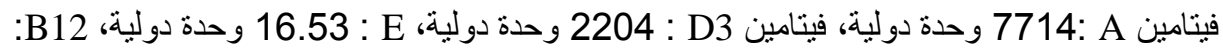

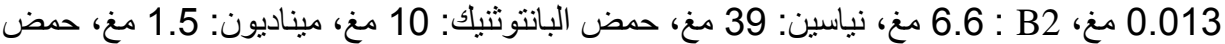

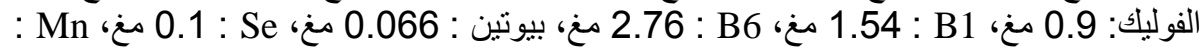

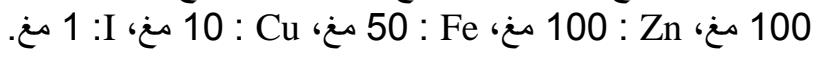

جدول رقم 2: تركيب الخلطات العلفية في المرحلة الثانية

\begin{tabular}{|c|c|c|c|c|}
\hline مثيو نين نباتي & الاصطناعي 2 & مثيونين نباتي & الاصطناعي 1 مثيو & مكونات الخلطة \\
\hline 66.35 & 66.3 & 66.37 & 66.37 & ذرة صفر اء \\
\hline
\end{tabular}


Assiut Vet. Med. J. Vol. 57 No. 130 July 2011

\begin{tabular}{|c|c|c|c|c|}
\hline 29.43 & 29.39 & 29.46 & 29.46 & كسبة الصويا \\
\hline 0.55 & 0.55 & 0.55 & 0.55 & زيت الصويا \\
\hline 1.7 & 1.7 & 1.7 & 1.7 & فوسفات ثنائية الكالسيوم \\
\hline 1.15 & 1.15 & 1.15 & 1.15 & كربونات الكالسيوم \\
\hline 0.15 & 0.15 & 0.1 & 0.1 & مثيونين حر \\
\hline 0.17 & 0.26 & 0.17 & 0.17 & كولين 60\% \\
\hline 0.1 & 0.1 & 0.1 & 0.1 & خلطة فيتامينات* \\
\hline 0.1 & 0.1 & 0.1 & 0.1 & خلطة معادن* \\
\hline 0.3 & 0.3 & 0.3 & 0.3 & ملح طعام \\
\hline 100 & 100 & 100 & 100 & المجموع \\
\hline
\end{tabular}

(*) 1 كل كن من العلف الجاهز يحقي على الفيتامينات و المعادن النادرة الآتية:

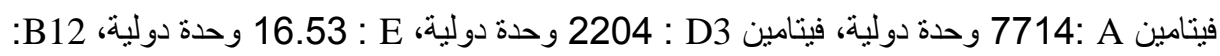

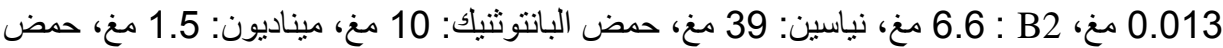

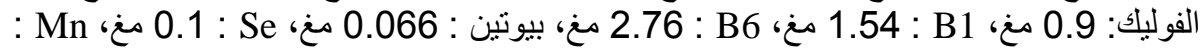

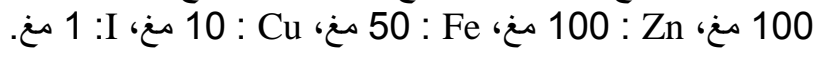

جدول رقم 3: القيم الغذائية للخلطات العلفية في المرحلة الأولى

\begin{tabular}{|c|c|c|c|c|}
\hline نباتي نين 2 & الاصطناعي 2 & نباتيونين 1 & الاصطناعي 1 مثيونين & \\
\hline 139.1 & 139.2 & 139.1 & 139.1 & $C / P$ \\
\hline 2894 & 2891 & 2891 & 2891 & طاقة قابلة للتمثيل ك.ك/كغ \\
\hline
\end{tabular}


Assiut Vet. Med. J. Vol. 57 No. 130 July 2011

\begin{tabular}{|c|c|c|c|c|}
\hline 20.79 & 20.76 & 20.77 & 20.77 & البروتين \% \\
\hline 1.13 & 1.13 & 1.13 & 1.13 & لايسين \% \\
\hline 0.63 & 0.63 & 0.52 & 0.52 & المثيونين\% \\
\hline 0.98 & 0.98 & 0.87 & 0.87 & \% م + ( ) \\
\hline 1 & 1 & 1 & 1 & كالسيوم \% \\
\hline 0.41 & 0.41 & 0.41 & 0.41 & فوسفور متاح \% \\
\hline 0.18 & 0.18 & 0.18 & 0.18 & صوديوم \% \\
\hline 0.29 & 0.29 & 0.29 & 0.29 & كلور \% \\
\hline 4.2 & 4.2 & 4.2 & 4.2 & الياف خام \% \\
\hline
\end{tabular}

جدول رقم 4: القيم الغذائية للخلطات العلفية في المرحلة الثانية

\begin{tabular}{|c|c|c|c|c|}
\hline نباتي 2 & الاصطناعي 2 & نباتي نين 1 & الاصطناعي 1 & \\
\hline 160.1 & 160.1 & 160.1 & 160.1 & $C / P$ \\
\hline 2943 & 2941 & 2942 & 2942 & طاقة قابلة للتمثيل ك.ك/كغ \\
\hline 18.38 & 18.36 & 18.37 & 18.37 & البروتين \% \\
\hline 96 & 0.96 & 0.96 & 0.96 & لايسين \% \\
\hline 0.43 & 0.43 & 0.39 & 0.39 & المثيونين\% \\
\hline 0.75 & 0.75 & 0.7 & 0.7 & \% (م + (م) \\
\hline 0.96 & 0.96 & 0.96 & 0.96 & كالسيوم \% \\
\hline 0.35 & 0.35 & 0.35 & 0.35 & فوسفور متاح \% \\
\hline 0.13 & 0.13 & 0.13 & 0.13 & صوديوم \% \\
\hline 0.22 & 0.22 & 0.22 & 0.22 & كلور \% \\
\hline 3.81 & 3.81 & 3.82 & 3.82 & الياف خام \% \\
\hline
\end{tabular}

جدول رقم 5: منوسط الوزن الحي الأسبوعي(غ) و الانحراف المعياري ونسبة النفوق

\begin{tabular}{|c|c|c|c|c|}
\hline مثيونين نباتي 2 & الاصطناعي 2 & مثيونين نباتي 1 & الاصطناعي 1 & الأسبوع المجموع \\
\hline $\begin{array}{c}\text { a } 160.18 \\
\pm 9.907\end{array}$ & $\begin{array}{r}\text { b } 154.83 \\
\pm 11.497\end{array}$ & $\begin{array}{r}156.85 \\
\pm 11.828\end{array}$ & $\begin{array}{l}\text { a } 159.80 \\
\pm 14.281\end{array}$ & الأول \\
\hline $\begin{array}{r}416.09 \\
\pm 27.245\end{array}$ & $\begin{array}{l}\text { b } 411.21 \\
\pm 31.793\end{array}$ & $\begin{array}{r}415.29 \\
\pm 29.429\end{array}$ & $\begin{array}{l}\text { a } 426.98 \\
\pm 33.178\end{array}$ & ني \\
\hline
\end{tabular}


Assiut Vet. Med. J. Vol. 57 No. 130 July 2011

\begin{tabular}{|c|c|c|c|c|}
\hline $\begin{array}{c}826.32 \\
\pm 51.274\end{array}$ & $\begin{array}{c}834.26 \\
\pm 54.265\end{array}$ & $\begin{array}{c}828.5 \\
\pm 61.688 \\
\end{array}$ & $\begin{array}{r}842.86 \\
\pm 73.520\end{array}$ & الثالث \\
\hline $\begin{array}{r}1270.8 \\
\pm 102.86\end{array}$ & $\begin{array}{r}1282.0 \\
\pm 76.908\end{array}$ & $\begin{array}{c}1301.1 \\
\pm 127.66\end{array}$ & $\begin{array}{r}1284.3 \\
\pm 125.71\end{array}$ & الر ابع \\
\hline $\begin{array}{r}1730.0 \\
\pm 159.70\end{array}$ & $\begin{array}{r}1743.9 \\
\pm 178.42\end{array}$ & $\begin{array}{r}1772.9 \\
\pm 201.34\end{array}$ & $\begin{array}{r}1730.7 \\
\pm 157.02\end{array}$ & ال \\
\hline $\begin{array}{r}2098.3 \\
\pm 198.97\end{array}$ & $\begin{array}{c}2129.7 \\
\pm 198.64\end{array}$ & $\begin{array}{c}2126.8 \\
\pm 233.69\end{array}$ & $\begin{array}{r}2075.9 \\
\pm 212.78\end{array}$ & السادس \\
\hline $1.61 \%$ & $2.98 \%$ & $1.56 \%$ & $3.17 \%$ & النفوق \% \\
\hline
\end{tabular}

ملاحظة: الأحرف المختلفة في نفس السطر و عند كل عمر تدل على وجود فروق معنوية على مستوى
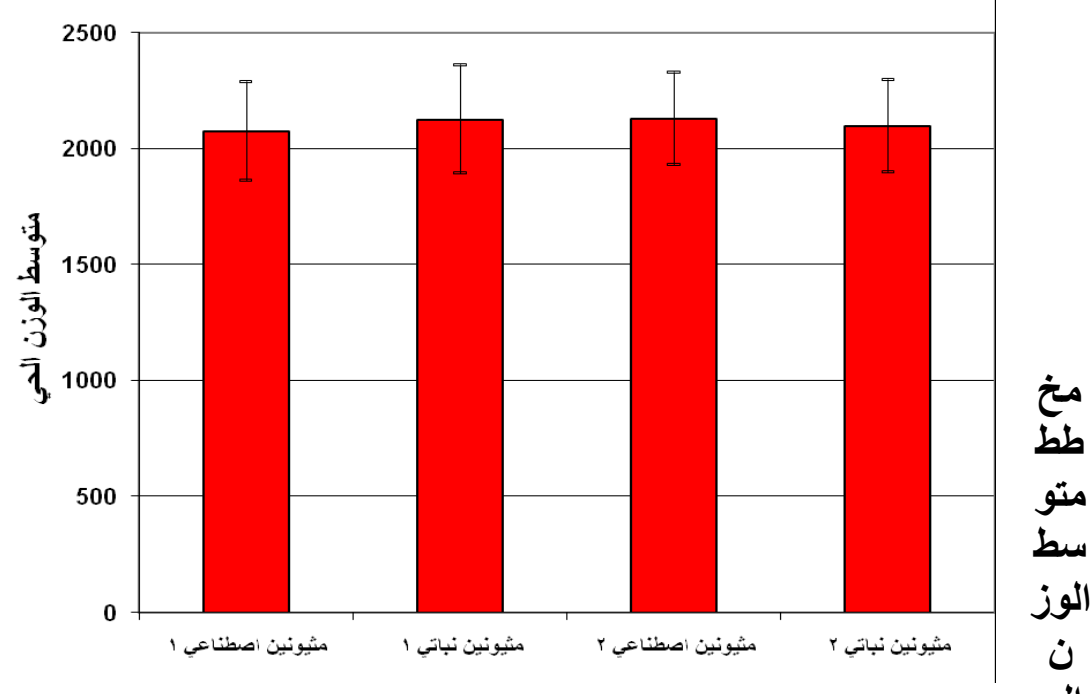

ي بعمر 6 أسابيع مع الانحراف المعياري 
Assiut Vet. Med. J. Vol. 57 No. 130 July 2011

جدول رقم 6: العلف المستهلك الأسبوعي و التر اكمي (غ/طير).

\begin{tabular}{|c|c|c|c|c|}
\hline مثيونين نباتي & الاصطناعي مثيونين 2 & مثيونين نباتي 1 & الاصطناعي 1 مثيونين & الأسبوع \\
\hline 137 & 127 & 132 & 138 & الأول \\
\hline 375 & 369 & 357 & 375 & الثناني \\
\hline 613 & 611 & 608 & 610 & الثالث \\
\hline 868 & 862 & 864 & 860 & الر ابع \\
\hline 1000 & 1018 & 995 & 1012 & الخامس \\
\hline 1032 & 1069 & 1031 & 1008 & السادس \\
\hline 1125 & 1107 & 1097 & 1123 & المرحلة الأولى \\
\hline 2900 & 2949 & 2890 & 2880 & المرحلة الثانية \\
\hline 4025 & 4056 & 3987 & 4003 & العلف التز اكمي \\
\hline
\end{tabular}

جدول رقم 7: معامل التحويل العلفي الأسبو عي و التر اكمي

\begin{tabular}{|c|c|c|c|c|}
\hline نباتي 2 & الاصطناعي مثيونين 2 & نباتي نين 1 & الاصطناعي 1 & الأسبوع \\
\hline 1.18 & 1.16 & 1.18 & 1.19 & الأول \\
\hline 1.46 & 1.44 & 1.38 & 1.4 & الثاني \\
\hline 1.49 & 1.44 & 1.47 & 1.47 & الثالث \\
\hline 1.95 & 1.93 & 1.83 & 1.95 & الر ابع \\
\hline 2.18 & 1.20 & 2.11 & 2.27 & الخامس \\
\hline 2.80 & 2.77 & 2.91 & 2.92 & السادس \\
\hline 1.92 & 1.90 & 1.87 & 1.93 & معامل التحويل العلفي \\
\hline
\end{tabular}

جدول رقم 8: تركيب الذبيحة ونسبة التصافي مع الانحر اف المعياري بعمر 42 يوم 
Assiut Vet. Med. J. Vol. 57 No. 130 July 2011

\begin{tabular}{|c|c|c|c|c|}
\hline ن مباتيونين 2 & الاصطناعي 2 & ن منيونين & الاصطناعي 1 & الأسبوع المجموعة \\
\hline $\begin{aligned} & 2085 \\
\pm & 54.314\end{aligned}$ & $\begin{array}{c}2268.8 \\
\pm 130.73\end{array}$ & $\begin{array}{r}2207.5 \\
\pm 259.02\end{array}$ & $\begin{aligned} & 2125 \\
\pm & 157.80\end{aligned}$ & الوزن الحي \\
\hline $\begin{array}{c}77.415 \\
\pm 1.9483\end{array}$ & $\begin{array}{c}77.801 \\
\pm 1.5419\end{array}$ & $\begin{array}{c}77.141 \\
\pm 2.2402\end{array}$ & $\begin{array}{c}76.948 \\
\pm 3.0906\end{array}$ & التصافي \% \\
\hline $\begin{array}{c}22.449 \\
\pm 1.7703\end{array}$ & $\begin{array}{c}22.782 \\
\pm 2.7681\end{array}$ & $\begin{array}{c}22.099 \\
\pm 4.8060\end{array}$ & $\begin{array}{c}21.587 \\
\pm 3.2146\end{array}$ & لحم الصدر \% \\
\hline $\begin{array}{c}18.344 \\
\pm 0.4723\end{array}$ & $\begin{array}{c}17.702 \\
\pm 2.1150\end{array}$ & $\begin{array}{c}17.163 \\
\pm 2.8734\end{array}$ & $\begin{array}{c}18.644 \\
\pm 3.3633\end{array}$ & لحم الفخذ \% \\
\hline $\begin{array}{c}40.793 \\
\pm 2.0362\end{array}$ & $\begin{array}{c}40.484 \\
\pm 4.8510\end{array}$ & $\begin{array}{c}39.262 \\
\pm 7.3943\end{array}$ & $\begin{array}{c}40.231 \\
\pm 6.4863\end{array}$ & مجموع لحم الصدر و الفذذ \\
\hline
\end{tabular}

ملاحظة: الأحرف المختلفة في نفس السطر و عند كل عمر تدل على وجود فروق معنوية على مستوى

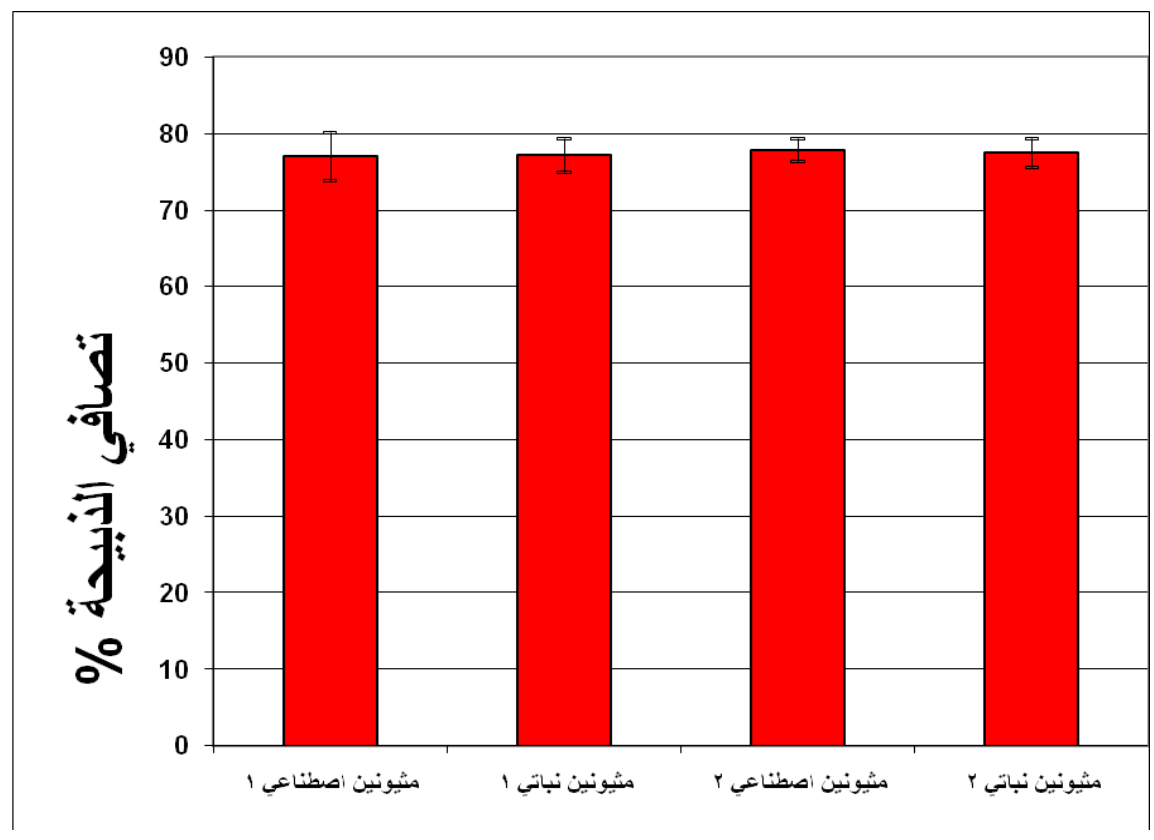

مخطط النسبة المئوية لتصافي الأبيحة مع الانحراف المعياري 


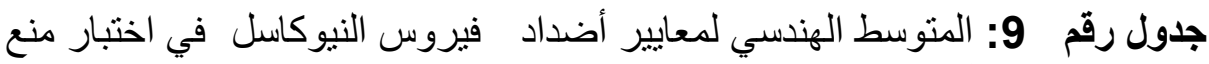

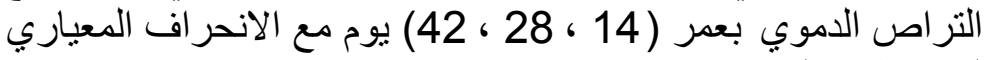
لمتوسطات القيم التري

\begin{tabular}{|c|c|c|c|c|}
\hline مثباتي 2 & الاصطناعي 2 & نباتي 1 نين & الاصطناعي 1 مثنين & الأسبوع المجموعة \\
\hline $\begin{array}{c}9.19 \\
\pm 5.36\end{array}$ & $\begin{array}{r}9.19 \\
\pm 5.36\end{array}$ & $\begin{array}{r}9.19 \\
\pm 3.57\end{array}$ & $\begin{array}{c}8 \\
\pm 0.0\end{array}$ & الثاني \\
\hline $\begin{array}{c}8 \\
\pm 4.38\end{array}$ & $\begin{array}{c}8 \\
\pm 4.38\end{array}$ & $\begin{array}{l}6.96 \\
\pm 6.0\end{array}$ & $\begin{array}{c}8 \\
\pm 6.0\end{array}$ & الر ابع \\
\hline $\begin{array}{c}21.1 \\
\pm 22.34\end{array}$ & $\begin{array}{c}21.1 \\
\pm 22.34\end{array}$ & $\begin{array}{c}16 \\
\pm 8.76\end{array}$ & $\begin{array}{r}13.93 \\
\pm 9.79\end{array}$ & السادس \\
\hline
\end{tabular}

ملاحظة: الأحرف المختلفة في نفس السطر و عند كل عمر تدل على وجود فروق معنوية على مستوى $\% 5$

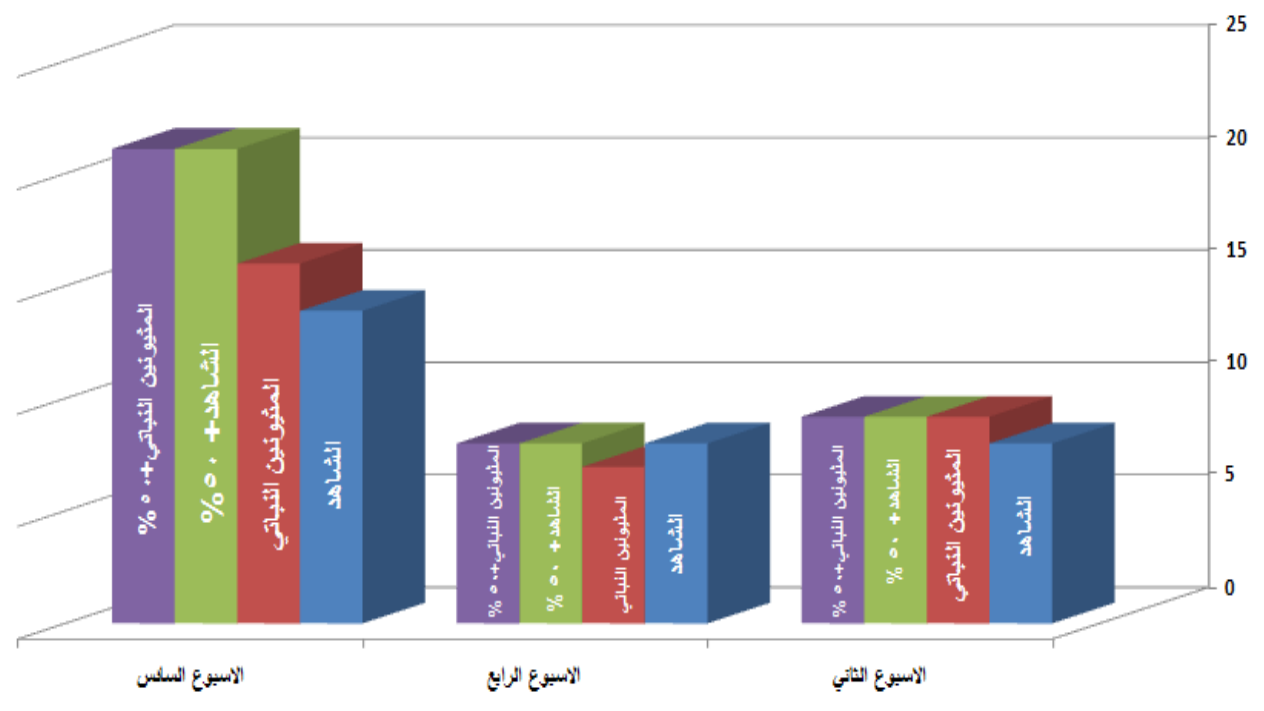

مخطط المتوسط الهندي لمعايير أضداد فيروس النيوكاسل 


\section{DISCUSSION \\ المناقشــة}

النتائج موضحة في الجداول (5 ، 6 ، 7 ،8 ،9).

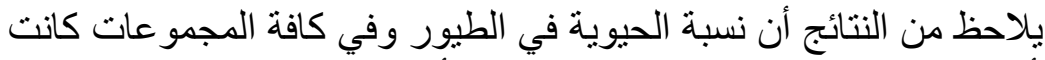

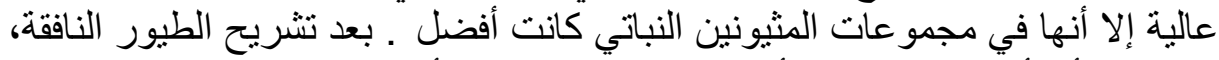

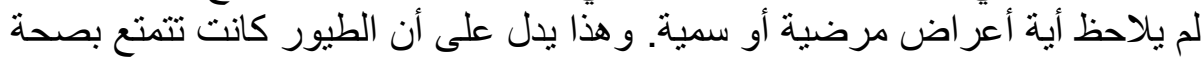
جيدة طيلة فترة التجربة.

النتائج المتعلقة بسر عة النمو (الجدول 5) تشير إلى عدم وجود فو ارق معنوية

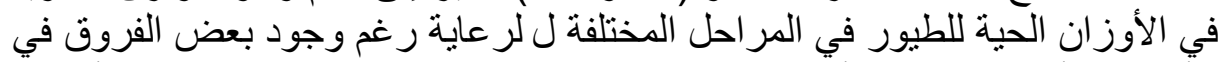

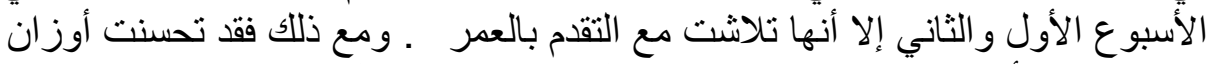

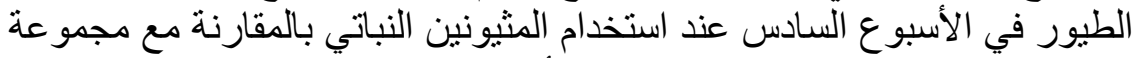

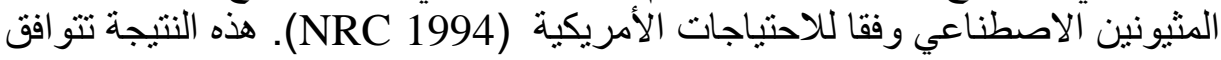

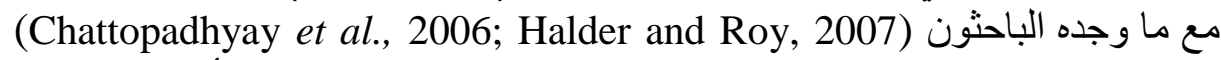

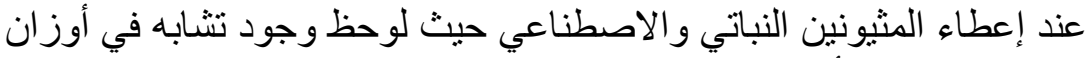

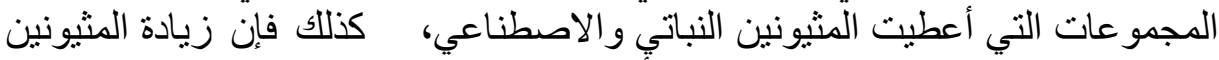

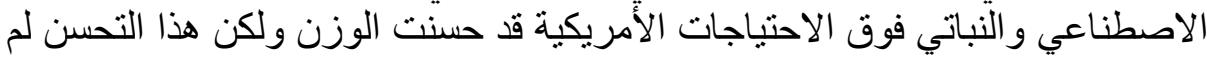

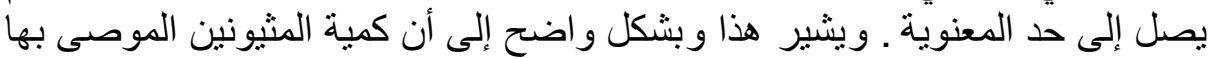

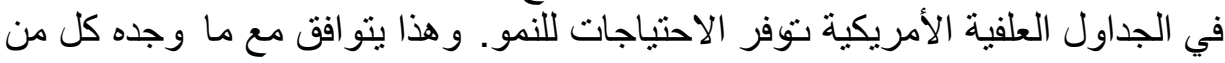
Hickling et al, (1990); Moran (1994); Schutte and Pack (1995b); بأنه ليس لزيـادة المثيونين فوق الاحتياجات الأمريكية

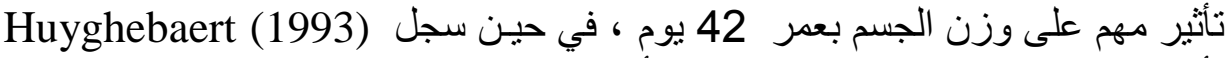

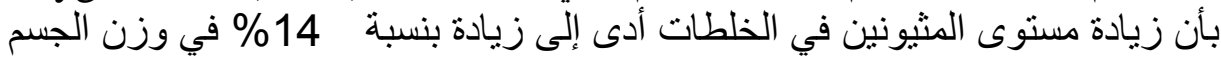
المكتسب بالمقارنة مع خلطات علفية فقيرة بالمثيونين.

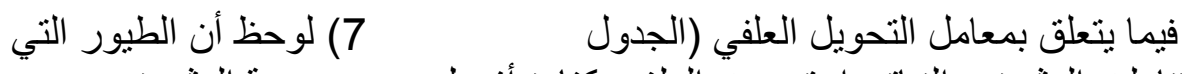

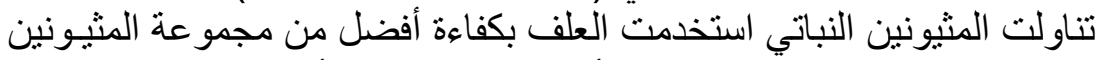

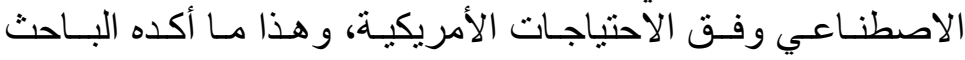

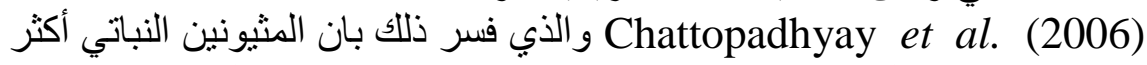

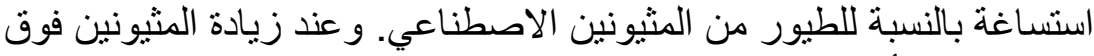

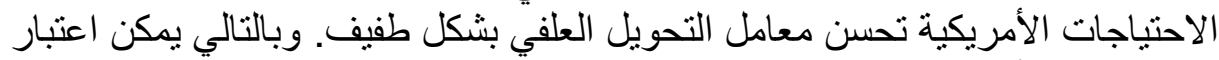

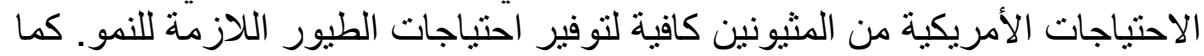

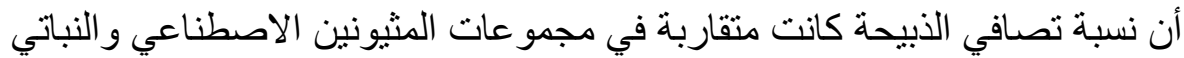

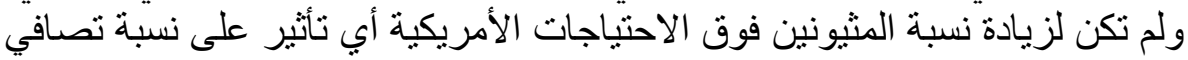

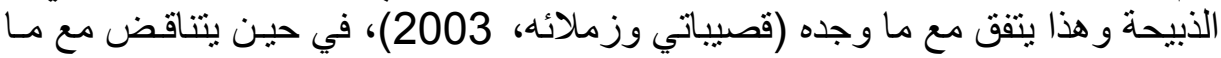

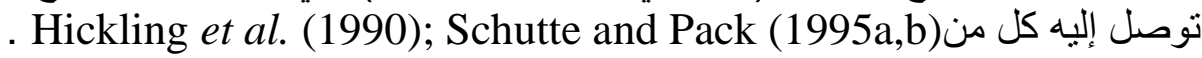


كما لم تلاحظ فروق بين معدل أضداد فيروس النيوكاسل بين المجمو عات

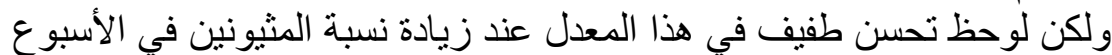

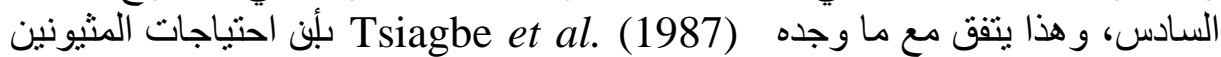
من أجل أفضل معيار للأجسام المضادة كان اكبر مما هو للنمو.

\section{CONCLUSIONS}

الاستنتاجات

تتلخص نتائج هذا البحث كما يلي:

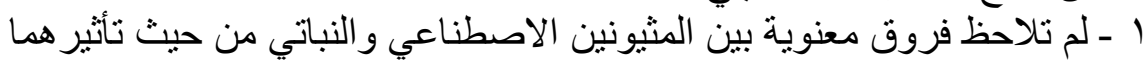
على سر عة النمو وكمية العلف المستهلكة ومعامل التهن التحويل العلفي وتركيب

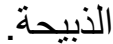

r - زيادة نسبة المثيونين فوق الاحتياجات الأمريكية ليس لها أي تأثثر يذكر على الإنى الكفاءة الإنتاجية للفروج.

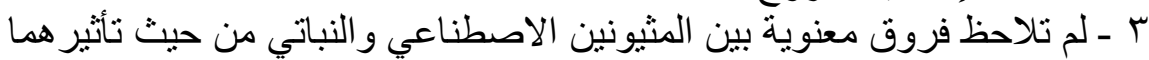

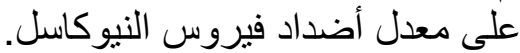
ع - زيادة نسبة المثيونين فوق الاحتياجات الأمريكية ليس لها أبي تأثير يذكر على النيل معدل أضداد فيروس النيوكاسل. ه ـ يمكن استخدام المثيونين النباتي كبديل عن المثيونين الاصني الصنطاعي في الخلطات العلفية المقدمة للفروج وخصوصنا أنه من مصني عن المنيادر طبيعية.

\section{REFERENCES المراجع العلمية}

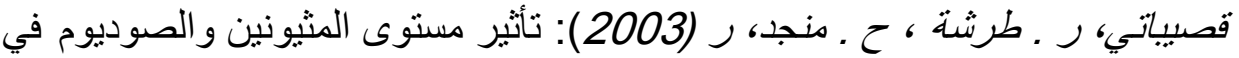

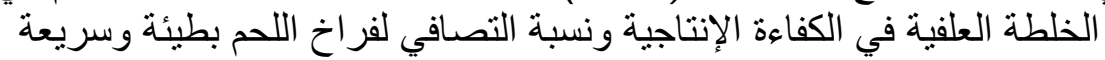
الترييش. مجلة جامعة البعث، 25: 103-120.

Butcher, G.D. and Miles, R.D. (2002): Interrelationship of Nutrition and Immunity .http:// www .edis.ifas.ufl.edu.

Chattopadhyay, K.; Mondal, M.K and Roy, B. (2006): Comparative efficacy of DL-methionine and herbal methionine on performance of broiler chicken. International Journal of Poultry Science 5 (11): 1034-1039.

Daljeet Kaur; Sodhi Sandeep; Dwivedi, P.N. and Nagra, S.S. (2007): Proceed. XXIV IPSACON, 25-27 ${ }^{\text {th }}$ April, Ludhiana: 94:511 and 12 
Doug, K. and Kirk, K. (2004): Influence of nutrition on immune status of the bird. Proceedings of the $24^{\text {th }}$ Technical Turkey Conference. P43.

Fancher, B.I. and Jensen, L.S. (1989): Influence on performance of three to six weeks old broilers of varying dietary protein contents with supplementation of essential amino acid requirements. Poult. Sci., 68: 113-123.

Halder, G. and Roy, B. (2007): Effect of herbal or synthetic methionine on performance, cost benefit ratio, meat and feather quality of broiler chicken. International Journal of Agricultural Research 2(12): 987-996.

Hickling, D.; Guenter, W. and Jackson, M.E. (1990): The effects of dietary methionine and lysine on broiler chicken performance and breast meat yield. Can. J. Anim. Sci., 70: 673-678.

Huyghebaert, G. and Pack, M. (1996): Effects of dietary protein content, addition of nonessential amino acids and dietary methionine to cysteine balance on responses to dietary sulphur-containing amino acids in broilers. Br. Poult. Sci., 37: 623-639.

Kalinowski, A.; Moran, E.T. Jr. and Wyatt, C. (2003a): Methionine and cystine requirements of slow- and fast-feathering male broilers from zero to three weeks of age. Poult. Sci., 82: 1423-1427.

Kalinowski, A.; Moran, E.T. Jr. and Wyatt, C.L. (2003b): Methionine and cystine requirements of slow- and fast-feathering male broilers from three to six weeks of age. Poult. Sci., 82: 1428-1437.

Konashi, S.; Takahashi, K. and Akiba, Y. (2000): Effects of dietary essential amino acid deficiencies on immunological variables in broiler chickens, Br. J.Nutr., 83: 499-456.

Lu, H. (2007): Avian Virology Diagnostic Protocols and Procedure. (Standerd Operation Procedure-SOPs). Preparationfor Avian Influenza Laboratory Consultancy. Under Fao and Usaid.

Maiorka, A.; Magro, N.; Bartels, H.A.; Kessler, A.M. and Penz, A.M. (2004): Different sodium levels and electrolyte balances in pre statter diet s for broilers. Brazilian J.Poult.Sci., 6: 143-146.

Moran, E.T., JR. (1994): Responses of broiler strains differing in body fat to inadequate methionine: Live performance and processing yields. Poult. Sci., 73:1116-1126.

National Research Council (1994): Nutrient Requirements of Poultry. $9^{\text {th }}$ rev. ed. Natl. Acad. Press, Washington, DC. 
North, M.O. and Bell, D.D. (1990): Commercial Chicken Production Manual. $4^{\text {th }}$ Edn.

Office International des Epizooties "OIE" (2002): Manual of Standards for Diagnostic Tests and Vaccines, Website:

Schutte, J.B. and Pack, M. (1995a): Sulfur amino acid requirement of broiler chicks from fourteen to thirty eight days of age. 1. Performance and carcass yield. Poult. Sci. 74: 480-487. http://www.oie.int/eng/normes/mmanual/A_summry.htm.

Schutte, J.B. and Pack, M. (1995b): Effects of dietary sulfur containing amino acids on performance and breast meat deposition of broiler chicks during the growing and finishing phases. Br. Poult. Sci. 36: 747-762.

Statistix Statistical 1.0 (1996): Analytical Computer Software Version 1.0 .

Tsiagbe, V.K.; Cook, M.E.; Harper, A.E. and Sunde, M.L. (1987a): Efficacy of cysteine in replacing methionine in the immune responses of broiler chickens. Poult. Sci., 66: 1138-1146. 